\title{
Plasma conditions for improved energy coupling into the gain region of the Ni-like Pd transient collisional x-ray laser
}

\author{
R. F. Smith, ${ }^{1}$ J. Dunn, ${ }^{1}$ J. Filevich, ${ }^{2}$ S. Moon, ${ }^{1}$ J. Nilsen, ${ }^{1}$ R. Keenan, ${ }^{1}$ V. N. Shlyaptsev, ${ }^{3}$ J. J. Rocca, ${ }^{2}$ \\ J. R. Hunter, ${ }^{1}$ and M. C. Marconi ${ }^{4}$ \\ ${ }^{1}$ Lawrence Livermore National Laboratory, Livermore, California 94551, USA \\ ${ }^{2}$ NSF ERC for Extreme Ultraviolet Science and Technology and Department of Electrical and Computer Engineering, \\ Colorado State University, Fort Collins, Colorado 80523, USA \\ ${ }^{3}$ University of California Davis-Livermore, Livermore, California 94551, USA \\ ${ }^{4}$ Physics Department, University of Buenos Aires, Argentina
}

(Received 8 October 2004; published 9 September 2005)

\begin{abstract}
We have directly probed the conditions in which the Ni-like Pd transient collisional x-ray laser is generated and propagates by measuring the near-field image and by utilizing picosecond resolution soft $\mathrm{x}$-ray laser interferometry of the preformed Pd plasma gain medium. The electron density and gain region of the plasma have been determined experimentally and are found to be in good agreement with simulations. We observe a strong dependence of the laser pump-gain medium coupling on the laser pump parameters. The most efficient coupling occurs with the formation of lower density gradients in the preformed plasma and when the duration of the main heating pulse is comparable to the gain lifetime ( $\sim 10 \mathrm{ps}$ for mid- $Z$ Ni-like schemes). This increases the output intensity by more than an order of magnitude relative to the commonly utilized case where the same pumping energy is delivered within a shorter heating pulse duration $(<3 \mathrm{ps})$. In contrast, the higher intensity heating pulses are observed to be absorbed at higher electron densities and in regions where steep density gradients limit the effective length of the gain medium.
\end{abstract}

DOI: 10.1103/PhysRevE.72.036404

PACS number(s): 52.50.Jm, 42.55.Vc, 42.87.Bg, 52.70.La

A detailed understanding of the plasma that constitutes the gain medium is crucial for the development of efficient x-ray lasers. Use of the prepulse technique has allowed $\mathrm{x}$-ray lasers to achieve saturated output using many different elements for the lasing media [1]. However, even the best laser-pumped $\mathrm{x}$-ray lasers typically have an efficiency of $10^{-6}$. In the transient collisional excitation (TCE) scheme a low intensity long pulse preforms a plasma, which is allowed to expand and cool before being heated by a high intensity short pulse [2]. This short pulse, in some cases with subpicosecond duration, rapidly heats the plasma to generate a high gain coefficient, saturated x-ray laser output [3], and x-ray laser pulses as short as 2 ps [4]. In experiments reported on high power laser drivers the pulse duration of the short pulse generated by chirped pulse amplification (CPA) is in the range of $0.3-3$ ps [3-7]. It has been assumed to some extent that by maximizing the intensity of the main heating pulse the temperature, collisional pumping, and local gain coefficient will also be maximized. Under these conditions the lowest saturated wavelength currently demonstrated is $7.3 \mathrm{~nm}$ for Nilike $\mathrm{Sm}[5]$.

To improve the efficiency we need to better understand the laser-plasma coupling and plasma characteristics of the $\mathrm{x}$-ray laser media. In this paper we combine the techniques of near-field imaging with recently developed picosecond $\mathrm{x}$-ray laser interferometry [8] to characterize the lasing medium for a Ni-like Pd x-ray laser. It is observed that a combination of controlling and reducing the plasma density gradients while matching the duration of the main pumping pulse to the gain lifetime at a specific density optimizes the coupling efficiency. This increases the x-ray laser output by an order of magnitude over the case where the same pump- ing energy is delivered into a higher intensity, shorter pulse. In contrast, short duration $(<3 \mathrm{ps})$ heating pulses are shown to more effectively couple their energy at high electron densities where increased levels of refraction limit the gainlength product.

Two different experiments using Pd targets were conducted on the Compact Multipulse Terawatt (COMET), $1054 \mathrm{~nm}$ wavelength, table-top laser facility at LLNL [3]. Saturated output on the Ni-like $\mathrm{Pd}$ X-ray laser $4 d^{1} S_{0^{-}}$ $4{ }^{1} P_{1}$ transition at $14.68 \mathrm{~nm}$ was achieved with a combination of a $600 \mathrm{ps}$ long pulse $\left(1.5 \mathrm{~J}, \sim 10^{11} \mathrm{~W} \mathrm{~cm}^{-2}\right)$ and a short $(0.5-27 \mathrm{ps})$ pulse $\left(4 \mathrm{~J}, 5-0.1 \times 10^{14} \mathrm{~W} \mathrm{~cm}^{-2}\right)$. A $1.6 \mathrm{~cm} \times 140 \mu \mathrm{m}$ line focus employed in a traveling wave geometry was used to irradiate a $1.25 \mathrm{~cm}$ long polished $\mathrm{Pd}$ slab target at normal incidence. More details of the experimental geometry may be found in Ref. [3]. As the x-ray laser is generated in a traveling wave geometry the electron density through which the $\mathrm{x}$-rays propagate is effectively frozen over the measured $\sim 4$ ps pulse duration [9]. The near-field plane of the x-ray laser leaving the plasma column was imaged by a $11.75 \mathrm{~cm}$ focal length spherical multilayer mirror and relayed onto a charge-coupled device (CCD) camera with a magnification of $\sim 22$ times. This gave a twodimensional (2D) intensity map of the X-ray laser as a function of distance away from the target surface. The near-field pattern was recorded as a function of long pulse to short pulse delay $(\Delta t)$ from $0-1$ ns peak-to-peak, while the CPA pulse duration was varied from $0.5-27$ ps. In all cases the pumping energy was kept fixed.

The second experiment utilized the unique diagnostic capabilities of our $14.7 \mathrm{~nm}$ x-ray laser interferometer that accurately measures the $2 \mathrm{D}$ electron density maps of laser- 
produced plasmas [8]. The picosecond time scale and short wavelength output of the x-ray laser probe beam mitigates effects due to refraction, absorption, and motion blurring making this technique well-suited for accurately diagnosing the high density, mm-scale plasmas, which are characteristic of the X-ray laser gain medium. A $0.31 \mathrm{~cm} \times 140 \mu \mathrm{m}$ line focus was used to heat a $0.2 \mathrm{~cm}$ polished Pd target placed in one arm of the interferometer. The energy delivered in the line focus was $300 \mathrm{~mJ}$ in the same $600 \mathrm{ps}$ long pulse to match the $\sim 10^{11} \mathrm{~W} \mathrm{~cm}^{-2}$ irradiation conditions for the preformed plasma of the Pd x-ray laser. The x-ray laser interferometry probes longitudinally through the resultant $\mathrm{Pd}$ plasma. The output plane of the plasma is imaged via a $25 \mathrm{~cm}$ focal length $\mathrm{Mo} / \mathrm{Si}$ multilayer optic and relayed to a $\mathrm{CCD}$ camera $\sim 5.5 \mathrm{~m}$ away giving an average magnification of $\sim 22 \times$. The number of fringe shifts, $N_{\text {fringe }}$, is related to the electron density $n_{e}$ by $[10], N_{\text {fringe }} \cong\left(n_{e} L\right) /\left(2 n_{\text {crit }} \lambda\right)$, where $n_{\text {crit }}$ is the critical density for the probe wavelength $\lambda$ and $L$ is the plasma length. For the $0.2 \mathrm{~cm}$ target used, one fringe shift corresponds to an electron density of $\sim 7.6$ $\times 10^{19} \mathrm{~cm}^{-3}$. Using this technique the $2 \mathrm{D}$ electron density profile was measured at different delays relative to the peak of the $600 \mathrm{ps}$ plasma forming beam. Within the x-ray laser context these times can be understood as different long pulse to short pulse delays $(\Delta t)$. The information from the two experiments together with simulations can then be combined to give a more complete characterization and understanding of the Pd x-ray laser under these different pumping conditions.

Figures 1(a), 1(b), and 1(c) show the combined electron density derived from the interferograms and near-field intensity maps for a $6.7 \mathrm{ps}$ short pulse incident at $\Delta t$ of $200 \mathrm{ps,}$ $700 \mathrm{ps}$, and $1 \mathrm{~ns}$, respectively. The color table has been optimized for each image in order to enhance detail. The absolute integrated intensity values are shown in Fig. 3. It is apparent from these images that the dimension and location of the near-field pattern is influenced greatly by the evolving electron density profile at different delays. At $\Delta t$ of $200 \mathrm{ps,}$ the density contours normal to the target surface are tightly bunched, indicating steep density gradients. As a result the $\mathrm{x}$-ray laser beam is refracted away from the target and the plasma exit appears to be centered at a density of 2 $\times 10^{19} \mathrm{~cm}^{-3}$. 2D LASNEX [11] simulations predict that the region which contributes most effectively to the X-ray laser amplification lies at a higher electron density of $\sim 1-2$ $\times 10^{20} \mathrm{~cm}^{-3}$. In addition, density gradients exist parallel to the target surface and the x-ray laser beam is extended in this direction as a result. For a $\Delta t$ of 700 ps the density gradients normal and parallel to the target surface have relaxed reducing the level of refraction. The x-ray laser output is centered at $\sim 1 \times 10^{20} \mathrm{~cm}^{-3}$ and the spatial extent in the direction parallel to the target is reduced. If the plasma is allowed to expand still further $(\Delta t$ of $1 \mathrm{~ns})$ the $\mathrm{x}$-ray laser is centered at $\sim 1.6 \times 10^{20} \mathrm{~cm}^{-3}$.

Figure 2 shows the near-field pattern for a $0.5 \mathrm{ps}$ pulse incident at a delay $\Delta t$ of $700 \mathrm{ps}$ similar to the density conditions shown in Fig. 1(b). The x-ray laser output is centered at $\sim 5 \times 10^{19} \mathrm{~cm}^{-3}$ while for the same density conditions the lower intensity $6.7 \mathrm{ps}$ heating pulse generates an $\mathrm{x}$-ray laser output centered at $1 \times 10^{20} \mathrm{~cm}^{-3}$. The increased deflection

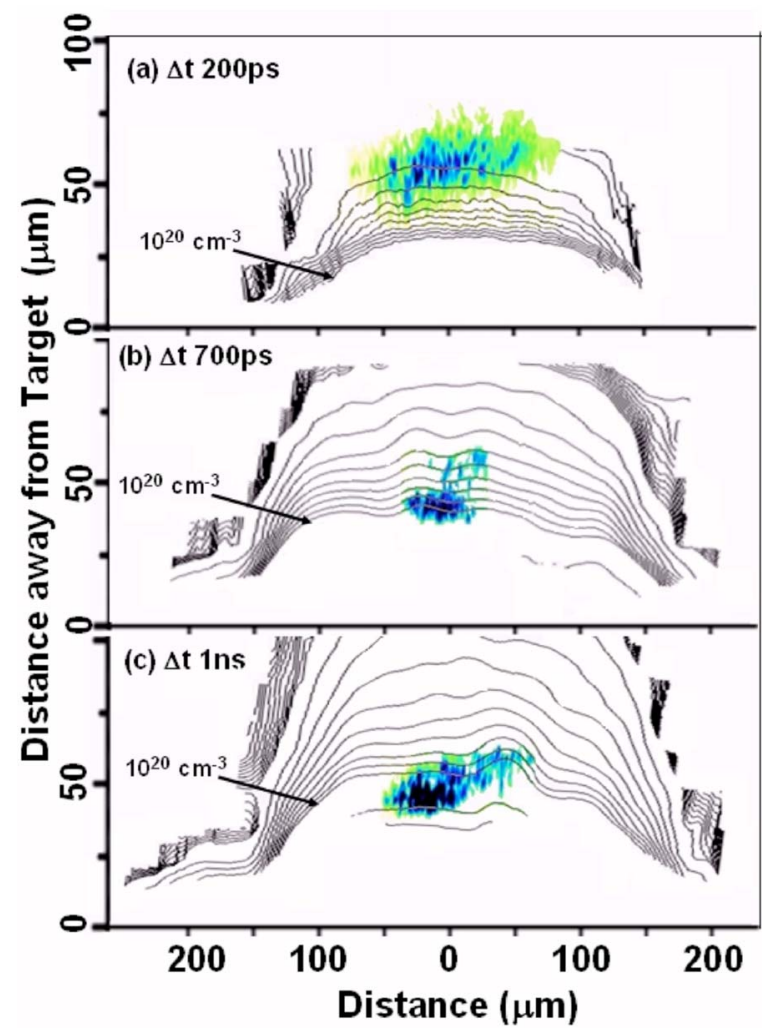

FIG. 1. (Color online) The near-field x-ray laser pattern is overlaid on interferometrically determined electron density maps for a short pulse duration of $6.7 \mathrm{ps}$ and for a $\Delta t$ of (a) $200 \mathrm{ps}$, (b) $700 \mathrm{ps,}$ and (c) $1 \mathrm{~ns}$. The extracted electron density contour map is shown in a logarithmic scale. The $10^{20} \mathrm{~cm}^{-3}$ contour is highlighted to indicate the approximate region where gain is predicted to occur. The color scales have been optimized for each particular image in order to enhance detail.

angle suggests that the region contributing most to the gain for the short CPA case is at a higher density, where larger density gradients exist, than for the $6.7 \mathrm{ps}$ CPA case. This is consistent with LASNEX simulations, which predict that the optimum temperature range of $T_{e} \sim 400-450 \mathrm{eV}$ to produce gain is generated at higher densities for the high intensity heating pulse. This also implies that the shorter pulse energy is absorbed more at higher densities. The greater refraction for the higher intensity pumping pulses cause the x-ray laser photons to be prematurely deflected out of the gain medium

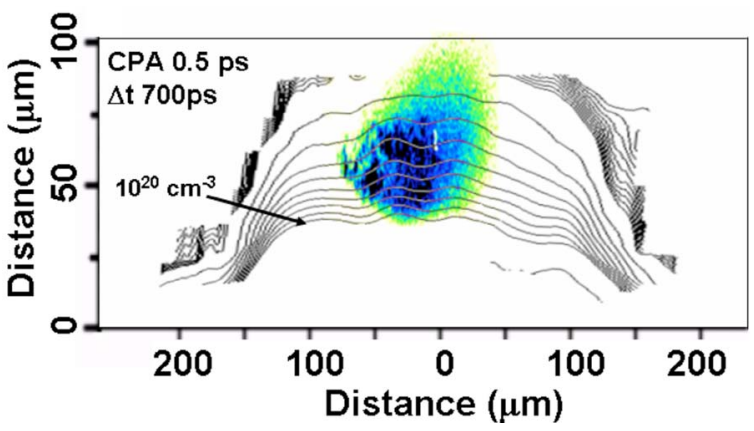

FIG. 2. (Color online) The near-field image and density map for a short pulse duration of $0.5 \mathrm{ps}$ and a $\Delta t$ of $700 \mathrm{ps}$. 


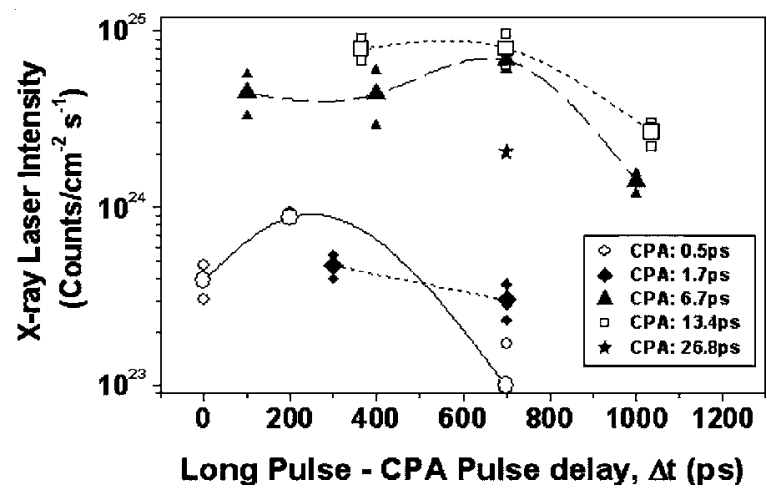

FIG. 3. X-ray laser output intensity as a function of different laser pumping parameters. The standard operating conditions for the $\mathrm{x}$-ray laser on the COMET laser facility is for a CPA pulse duration of $6.7 \mathrm{ps}$ with a long pulse to short pulse delay, $\Delta t$, of $700 \mathrm{ps}$. The large symbols represent the average of the individual shots (small symbols).

which results in reduced levels of amplification (see Fig. 3). This suggests that the pumping pulse durations $<3$ ps that are routinely used on large laser facilities are not optimum.

By integrating over the area of the footprint and taking into account the measured pulse durations [9] we can determine the relative output intensity of the x-ray laser as a function of laser pump parameters (Fig. 3). The highest output is observed for the main heating pulse durations of 6.7 and $13.4 \mathrm{ps}$ and for a $\Delta t$ of $\sim 700 \mathrm{ps}$. Under these pumping conditions previous measurements have determined the x-ray lasing transition to be saturated [12]. The outputs for the shorter duration CPA pulses (1.7 ps and $0.5 \mathrm{ps}$ ) are more than an order of magnitude less than for the 13 ps case and are considered to be unsaturated. It is possible to drive the shorter pulse driven $\mathrm{x}$-ray laser into saturation but it requires higher laser intensities [5-7]. We note on comparison with Fig. 1(b) and Fig. 2 that as the x-ray laser becomes more saturated the footprint becomes more concentrated in space, which is a result of the localized region of high gain dominating the amplification process. As the CPA pulse duration is increased to $\sim 27 \mathrm{ps}$ the $\mathrm{x}$-ray laser output intensity falls off from the peak value. For a given heating pulse duration there is a strong dependence on the $\mathrm{x}$-ray laser output as a function of $\Delta t$. The shorter duration heating pulses exhibit slightly enhanced output for reduced values of $\Delta t$. This is consistent with results for a Ni-like Sm x-ray laser which was optimized at $\Delta t$ of $\sim 150 \mathrm{ps}$ and pumped with a $1 \mathrm{ps}$ pulse [5]. As shown in Fig. 2, this is not a result of more effective beam propagation but is expected to be representative of the higher local gain coefficient for early $\Delta t$ (albeit more briefly sampled) due to the presence of higher temperatures and densities within the plasma. For the 6.7 ps heating pulse the more relaxed density gradients yield reduced levels of refraction and as a consequence the $\mathrm{x}$-ray laser photons spend longer within the gain region and experience greater amplification. At the image plane shown in Fig. 1(b) the $\mathrm{x}$-ray laser has traversed through the $12.5 \mathrm{~mm}$ long amplifying medium with very small deflection. From previous measurements we estimate the deflection angle of the x-ray laser under these pumping conditions to be $<5 \mathrm{mrad}$ [3]. Ray

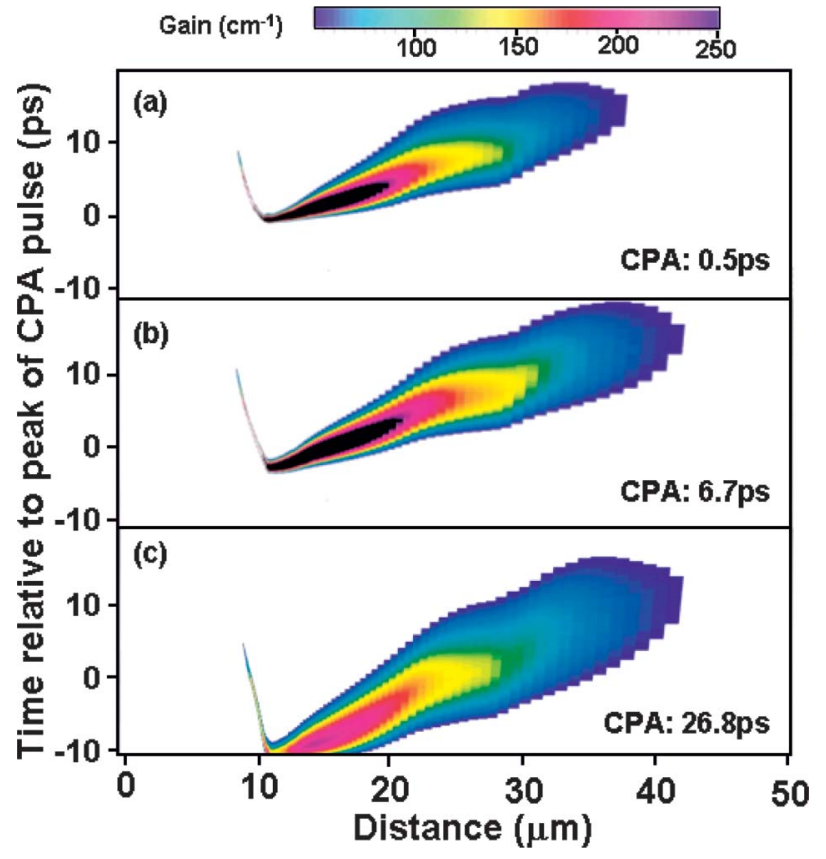

FIG. 4. (Color) 1D LASNEX simulations show the gain profile of the $4 d{ }^{1} S_{0} \rightarrow 4 p{ }^{1} P_{1}$ Ni-like Pd x-ray laser pumped with a short pulse duration of (a) $0.5 \mathrm{ps}$, (b) $6.7 \mathrm{ps}$, and (c) $27.8 \mathrm{ps}$ for a delay of $\Delta t$ of $700 \mathrm{ps}$ after the peak of the long pulse. The time axis is shown relative to the peak of the main heating pulse. The color table for gain saturates in the center of the contours at values of $250 \mathrm{~cm}^{-1}$.

tracing in 1D back through the experimentally determined density profile $(\Delta t \sim 700 \mathrm{ps})$ places the gain region at a density of $\sim 2 \times 10^{20} \mathrm{~cm}^{-3}$ at $20-25 \mu \mathrm{m}$ away from the target surface. These results offer the first experimental confirmation that the gain region in the TCE scheme originates at electron densities $>10^{20} \mathrm{~cm}^{-3}$. For a $\Delta t$ of $1 \mathrm{~ns}$ the output intensity has dropped by a factor of 7 over the 700 ps case (Fig. 3) even though the beam guiding is better, see Fig. 1. This is due to a drop in the electron temperature within the more expanded plasma.

LASNEX simulations of the irradiation conditions were used to estimate the average ionization within the gain region at the time of interaction of the CPA pulse $(\Delta t=0-0.7 \mathrm{~ns})$. The plasma was determined to be $3 \pm 1$ stages, i.e., Ga-like, below the Ni-like ion required for lasing. This indicated, for these prepulse conditions, the short pulse was required to ionize the plasma to the Ni-like ion in a few picoseconds as well as pump the inversion. Secondly, the actual electron density during the inversion was up to $35 \%$ higher in the $1-2 \times 10^{20} \mathrm{~cm}^{-3}$ density region than the electron density measured through interferometry of the prepulse plasma. The density gradients however were calculated to remain unchanged. Below $10^{20} \mathrm{~cm}^{-3}$ the electron density remained unperturbed by the short pulse interaction over the period in which gain takes place. It has been recently reported that the contribution from the bound electrons to the refractive index of a plasma can affect the determination of electron density using soft x-ray interferometry [13]. This effect on the interferometry of the Pd plasma was calculated to be less than $10 \%$ for the predicted ionization conditions. These factors 
were taken into account for measuring the density conditions in which the X-ray laser gain is generated and propagated.

To model these experiments the LASNEX calculations of densities and temperatures are used as input to the CRETIN code [14]. CRETIN calculates the gain profile as a function of distance away from the target surface and time relative to the peak of the short pulse for three different pulses incident at the same $\Delta t$ of $700 \mathrm{ps}$ after the peak of the long pulse laser (Fig. 4). The duration of the gain is calculated to be largely insensitive to the duration of the heating pulse. This is supported by recent measurements in which the output pulse durations change by only a factor of $2(\sim 4-8 \mathrm{ps})$ when the duration of the main heating pulse is changed by a factor of $55(0.5-27.8 \mathrm{ps})$ [9]. The predicted gain coefficient is higher and shorter lived close to the target surface due to the faster collisional rates at these higher electron densities. We can see from the contour plot in Fig. 4(a) that the peak of the gain in the plasma heated by the $0.5 \mathrm{ps}$ pulse occurs after the laser irradiation is switched off. The short pulse energy couples most effectively to the short lived and spatially small gain region close to the critical density where refraction effects eject the x-ray laser photons prematurely out of the gain medium (Fig. 2). For the lower intensity 6.7 ps pulse, shown in Fig. 4(b), the energy is coupled more effectively into the lower density gain region. Here the heating occurs at the same time and over the same time scale as the gain duration. The reduced density gradients allow for more effective beam propagation and as a result the x-ray laser output is enhanced by more than an order of magnitude over the high intensity 0.5 ps case. For the 27 ps pulse the gain has dissipated before most of the energy has been delivered on target. There- fore the coupling is poorly optimized and the x-ray laser output intensity drops relative to the $6.7 \mathrm{ps}$ case.

We have obtained insights into the dynamics within the gain region of the Ni-like $\mathrm{Pd} \mathrm{x}$-ray laser in a series of experiments combining the complimentary techniques of nearfield imaging and $\mathrm{x}$-ray interferometry. We infer from the increased levels of refraction that short heating pulses couple their energy most effectively at higher densities where large density gradients limit the effective length of the gain medium. By matching the duration of the short pulse $\sim 10 \pm 5$ ps with the gain duration $(\sim 10 \mathrm{ps})$ an enhancement in the $\mathrm{x}$-ray laser output intensity of more than an order of magnitude was observed over the commonly utilized short pulse pumping case $(<2 \mathrm{ps})$. Tailoring the laser pump duration for a given TCE scheme is expected to give a more efficient route towards saturated lasing within the water window $(2.33-4.37 \mathrm{~nm})$ where applications such as submicron biological imaging become viable [15].

The authors would like to thank Albert Osterheld for continued support of this work. This work was performed under the auspices of the U.S. Department of Energy by the University of California, Lawrence Livermore National Laboratory through the Institute of Laser Science and Application, under Contract No. W-7405-Eng-48. This research was sponsored by the National Nuclear Security Administration under the Stewardship Science Academic Alliances program through DOE Research Grant No. DEFG03-02NA00062. The CSU researchers also gratefully acknowledge the partial support of the NSF ERC Center for Extreme Ultraviolet Science and Technology, award number EEC-0310717.
[1] J. Nilsen et al., Phys. Rev. A 48, 4682 (1993).

[2] P. V. Nickles et al., Phys. Rev. Lett. 78, 2748 (1997).

[3] J. Dunn et al., Phys. Rev. Lett. 84, 4834 (2000).

[4] A. Klisnick et al., Phys. Rev. A 65, 033810 (2002).

[5] R. E. King et al., Phys. Rev. A 64, 053810 (2001).

[6] A. Klisnick et al., J. Opt. Soc. Am. B 17, 1093 (2000).

[7] H. Tang et al., Jpn. J. Appl. Phys., Part 1 42, 443 (2003).

[8] R. F. Smith et al., Phys. Rev. Lett. 89, 065004 (2002).

[9] J. Dunn et al., SPIE Int. Soc. Opt. Eng. Proc., Vol. 5197, edited by E. E. Fill and S. Suckewer, 2003, pp. 51-59.
[10] L. B. Da Silva et al., Phys. Rev. Lett. 74, 3991 (1995).

[11] G. B. Zimmerman and W. L. Kruer, Comments Plasma Phys. Controlled Fusion 2, 51 (1975).

[12] J. Dunn et al., J. Phys. IV 11, 2 (2001).

[13] J. Filevich et al. Phys. Rev. Lett. 94, 035005 (2005); J. Nilsen and J. H. Scofield, Opt. Lett. 29, 2677 (2004).

[14] H. A. Scott, J. Quant. Spectrosc. Radiat. Transf. 71, 689 (2001).

[15] J. C. Solem and G. C. Baldwin, Science 218, 229 (1982). 